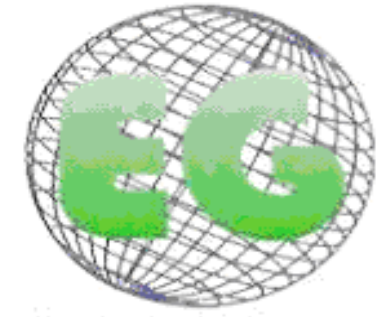

ISSN 1695-6141

N 24

\title{
Historia de la rehabilitación en Brasil, en el mundo y el papel de enfermería en este contexto: reflexiones y tendencias basadas en la revisión de literatura
}

História da reabillitação no Brasil, no mundo e o papel da enfermagem neste contexto: reflexões e tendências com base na revisão de literatura

\section{*Aparecida de Souza, L. **Mancussi e Faro, AC. \\ *Enfermera. Magister por la Escuela de Enfermería de la Universidad de São Paulo. **Profesora Doctora de la Escuela de Enfermería de la Universidad de São Paulo. Brasil.}

Palabras clave: Rehabilitación; Historia de la Enfermería; Políticas Públicas.

Palavras-chave: Reabilitação; História da Enfermagem; Políticas Públicas

Keywords: Rehabilitation, Nursing History, Public Policy.

\section{RESUMEN}

Se trata de un estudio de revisión bibliográfica, con el objetivo de describir los hechos históricos sobre la Rehabilitación en Brasil y en el Mundo así como de reflexionar sobre el papel de la enfermera en el contexto histórico de la rehabilitación. El interés mundial por la Rehabilitación surgió por cuatro acontecimientos históricos: dos Grandes Guerras, proceso acelerado de urbanización e industrialización, propagación de epidemias y aumento de accidentes de trabajo. En Brasil las instituciones de rehabilitación datan de periodos históricos diferentes y adoptan modelos de atención también diferentes. La enfermería comienza a construir su papel en rehabilitación a partir de 1944, con la creación del Centro de Traumatizados Medulares en la Gran Bretaña, y en los dias actuales es considerada miembro fundamental del equipo multiprofesional. Conocer la historia nos lleva a reflexionar sobre el contexto actual de la rehabilitación como la ciencia de la inclusión social, que lucha por tratamientos especializados y del establecimiento de políticas sociales y de salud para incluir todas las personas que presentan deficiencias.

\section{RESUMO}

Trata-se de um estudo de revisão bibliográfica, com objetivo de descrever os fatos históricos sobre a Reabilitação no Brasil e no Mundo e refletir sobre o papel da enfermagem no contexto histórico da reabilitação. O interesse mundial pela reabilitação ocorreu por quatro acontecimentos históricos: duas Grandes Guerras, processo acelerado de urbanização e industrialização, propagação de epidemias e aumento de acidentes de trabalho. No Brasil as instituições de reabilitação datam de períodos históricos diferentes e adotam modelos assistenciais também diferentes. A enfermagem começa a construir seu papel na reabilitação a partir de 1944, com a criação do Centro de Traumatizados Medulares na Grã-Bretanha, nos dias atuais é considerada membro fundamental da equipe multiprofissional. Conhecer a História nos leva a refletir e questionar sobre o contexto 
atual da reabilitação como a ciência da inclusão social, que luta por tratamentos especializados e de estabelecimento de políticas de saúde e sociais para incluir todas as pessoas que apresentam deficiências.

\section{ABSTRACT}

This is a review of the literature, in order to describe the historical facts about the rehabilitation in Brazil and around the world and reflect on the role of nursing in the historical context of rehabilitation. Global interest in rehabilitation of four historic events occurred: two world wars, accelerated urbanization and industrialization, spread of epidemics and increased accidents. In Brazil, rehabilitation institutions dating from different historical periods and take care models are also different. The nurse begins to build its role in rehabilitation from 1944, with the creation of the Traumatized Spinal Cord Center in Britain, is today considered a key member of the multidisciplinary team. Knowing the history leads us to reflect and inquire about the current context of rehabilitation science and social inclusion, fighting for specialized treatments and establishment of health and social policies to include all people with disabilities.

\section{INTRODUCCIÓN}

Hasta un pasado reciente los hospitales eran orientados en el sentido de la especialización y dentro del concepto restringido de la cura. El hospital no restablecía al paciente, de modo a establecer su bienestar, el de su familia y de su grupo social ${ }^{(1)}$.

Con el paso de los años la necesidad del trabajo integral volcado para el paciente se hizo fundamental, y así se creó la cuarta función de la medicina, después de la promoción, prevención y tratamiento médico propiamente dicho, nace la Rehabilitación ${ }^{(1)}$.

Reabilitación es un proceso educativo y de atención multiprofesional, que prima la búsqueda compartida del desarrollo de las capacidades remanentes, prevención del agravamiento de la incapacidad y de la aparición de complicaciones. Es compartida porque involucra al paciente, al cuidador familiar y al profesional especialista en rehabilitación.

Se sabe que el interés mundial por la Rehabilitación tuvo lugar principalmente por cuatro acontecimientos históricos: las dos grandes guerras mundiales, proceso acelerado de urbanización e industrialización lo que favoreció la propagación de epidemias y aumento de accidentes de trabajo ${ }^{(2)}$.

Así, nace a la necesidad de restituir esas personas, en relación a las capacidades individuales y sociales ${ }^{(1,2)}$.

En Brasil las instituciones de atención a las personas con deficiencias, datan de periodos históricos diferentes y adoptan modelos también diferentes de acuerdo con las circunstancias y agentes de su constitución, así como con el tipo de deficiencia que se quiere rehabilitar ${ }^{(3)}$.

En enfermería se considera el papel educativo y rehabilitador del enfermero desde el inicio de la Enfermería Moderna, cuando Florence Nightingale, en 1859, en la guerra de Crimea, prueba la eficiencia de las enfermeras capacitadas para la recuperación de los soldados heridos en la guerra ${ }^{(4)}$. 
Es a partir de ahí que la enfermería comienza a tener su papel mejor definido en este contexto, mostrando su importancia junto a esos servicios y procesos de rehabilitación.

\section{OBJETIVOS}

Describir los hechos históricos sobre la Rehabilitación en el Brasil y en el mundo según análisis de textos en forma de publicaciones.

Reflexionar sobre el papel de enfermería en el contexto histórico de la Rehabilitación en publicaciones impresas y en bases de datos electrónicos.

\section{METODOLOGÍA}

Este estudio se realizó mediante revisión bibliográfica. La muestra constó de textos, sin límite de tiempo, que se ocupan de la Historia de la Reabilitación y de la Enfermería en rehabilitación en el Brasil y en el Mundo.

La búsqueda sistematizada se realizó en libros, artículos de revistas cientificas y textos en bases electrónicas como los bancos de datos de MEDLINE y LILACS. Otra estrategia utilizada para identificar los estudios más antiguos fue extender la búsqueda a las referencias bibliográficas de los materiales seleccionados.

Para la localización fueron usados los siguientes descriptores de asuntos de la Biblioteca Virtual de Salud BVS/BIREME, en portugués, español e inglés: "historia de la rehabilitación, rehabilitación física, historia de la enfermería y enfermería"

Los criterios para la inclusión definidos fueron: artículos en portugués, español o inglés, libros o capítulos de libros, editoriales y textos encontrados en las bases de datos o citados en las referencias bibliográficas del material seleccionado, que tratan sobre la Historia de la Rehabilitación, o parte de ella y que tuviesen, también, datos culturales sobre la rehabilitación en el Brasil y en el Mundo, independiente de la categoría profesional que haya conducido el estudio y del referencial metodológico utilizado.

Para la colecta de datos y categorización sistematizada de las publicaciónes fue utilizado un instrumento (Anexo 1), que consta de: datos de identificación de la publicación (título del artículo, periódico, año de publicación y país de origen); datos sobre los autores (sexo y formación académica) y descriptores. El análisis de los textos fue hecho simultáneamente con la cumplimentación del instrumento de colecta y categorización de los datos.

\section{PRESENTACIÓN DE LOS RESULTADOS Y DISCUSIÓN.}

De un total de 38 textos seleccionados, 23 eran artículos, 10 libros o capítulos de libros textos, 3 textos extraídos de Internet y 2 editoriales de revistas que representaban partes del tema tratado. Después de la selección, se realizó la lectura exhaustiva de los textos para la caracterización y análisis del contenido..

Dentro los editoriales fueron encontrados solamente dos, publicados en 2006 y 2008 respectivamente, siendo ambos extraídos de un periódico brasileño no especializado en rehabilitación, pero que dedicó dos números especiales al tema. Editoriales éstos escritos por médicos siendo 4 del sexo masculino y 3 del sexo femenino. 
Entre los textos de Internet, todos son de sites brasileños y del total de 4 autores, 3 son mujeres y solo 1 hombre. 2 de las autoras pertenecen al area de educación y los demás no mencionan su área de actuación específica en los textos.

Fueron diez los libros texto o capítulos de libros seleccionados para la investigación, publicados entre los años 1963 y 2006. Dos específicos del área de enfermería, seis específicos en rehabilitación, uno especifico sobre fisioterapia y uno sobre rehabilitación y enfermería.

Los artículos publicados y seleccionados para esta investigación eran de entre 1959 y 2008, verificandose un total de 23.

El periodo de mayor publicación fue entre los años 2000 a 2008, como muestra la Tabla 1 abajo.

[Tabla 1. Distribución de los artículos según el periódico de publicación.]

\begin{tabular}{|c|c|}
\hline \multicolumn{2}{|c|}{$\begin{array}{c}\text { Distribución de los artículos según el } \\
\text { período de publicación }\end{array}$} \\
\hline Período & Porcentagem (\%) \\
\hline Década de 50 & 4,3 \\
\hline Década de 60 & 4,3 \\
\hline Década de 70 & 4,3 \\
\hline Década de 80 & 4,3 \\
\hline Década de 90 & 30,7 \\
\hline 2000 à 2008 & 52,3 \\
\hline
\end{tabular}

Con relación a la fuente de publicación de estos artículos, predominó un periódico nacional con el tema específico sobre rehabilitación, como muestra la Tabla 2.

[Tabla 2. Distribución del porcentaje de publicaciónes de los periódicos consultados, según el tema abordado.]

\begin{tabular}{|c|c|}
\hline $\begin{array}{c}\text { Distribución del porcentaje de publicaciónes de los periódicos } \\
\text { consultados, según el tema abordado }\end{array}$ \\
\hline Periódico & Porcentaje (\%) \\
\hline Texto e Contexto - Enfermagem & 12,9 \\
\hline Ciências e Saúde Coletiva & 4,3 \\
\hline Revista Brasileira de Enfermagem & $\mathbf{8 , 6}$ \\
\hline
\end{tabular}




\begin{tabular}{|c|c|}
\hline Rev. Gaúcha de Enfermagem & 4,3 \\
\hline Revista Latino Americana de Enfermagem & 8,6 \\
\hline Revista da Escola de Enfermagem da USP & 4.3 \\
\hline Revista Cubana de Enfermeria & 4.3 \\
\hline Rev. Paulista de Hospitais & 8,6 \\
\hline Reabilitation Nursing & 4,3 \\
\hline Gaceta Médica Caracas & 4,3 \\
\hline Acta Fisiátrica & 26 \\
\hline Revista de Saúde Pública & 4,3 \\
\hline O Mundo da Saúde & 4,3 \\
\hline
\end{tabular}

En lo relacionado al país de origen del periódico, hubo predominio de periódicos brasileños $76,9 \%$, los demás fueron de origen cubano, venezolano y americano, como se muestra en la Gráfico 1.

[Gráfico 1. Distribución de los periódicos según la nacionalidad de publicación.]

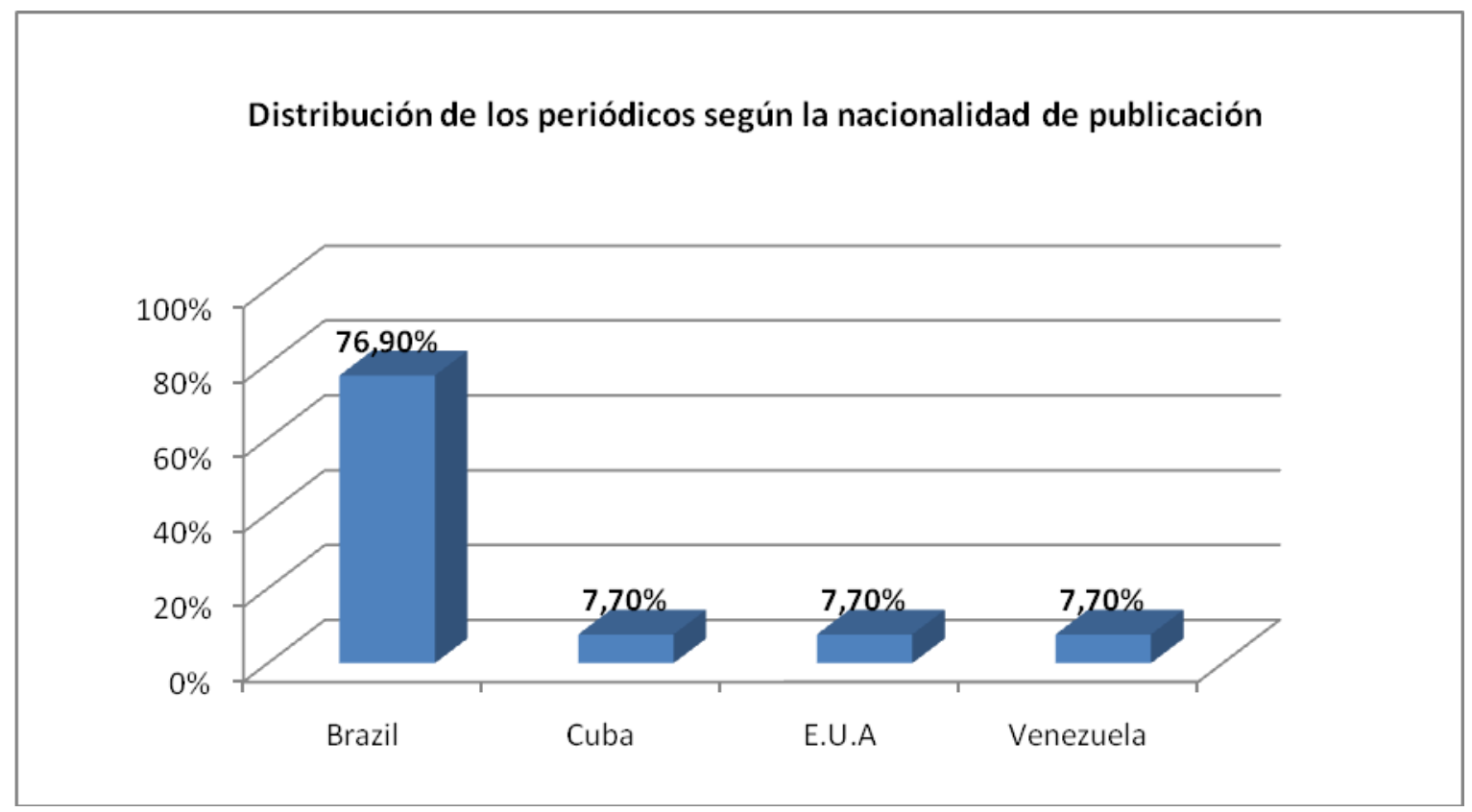

De los periódicos seleccionados para esta investigación, 46,2\% son específicos del área de enfermería, 38,4\% abordan contenidos generales de la salud, 7,4\% son específicos del área de rehabilitación y 7,4\% específicos de las dos áreas, enfermería y rehabilitación, como se muestra en el Gráfico 2. 
[Gráfico 2. Distribución de los periodicos según su especificidad de publicación.]

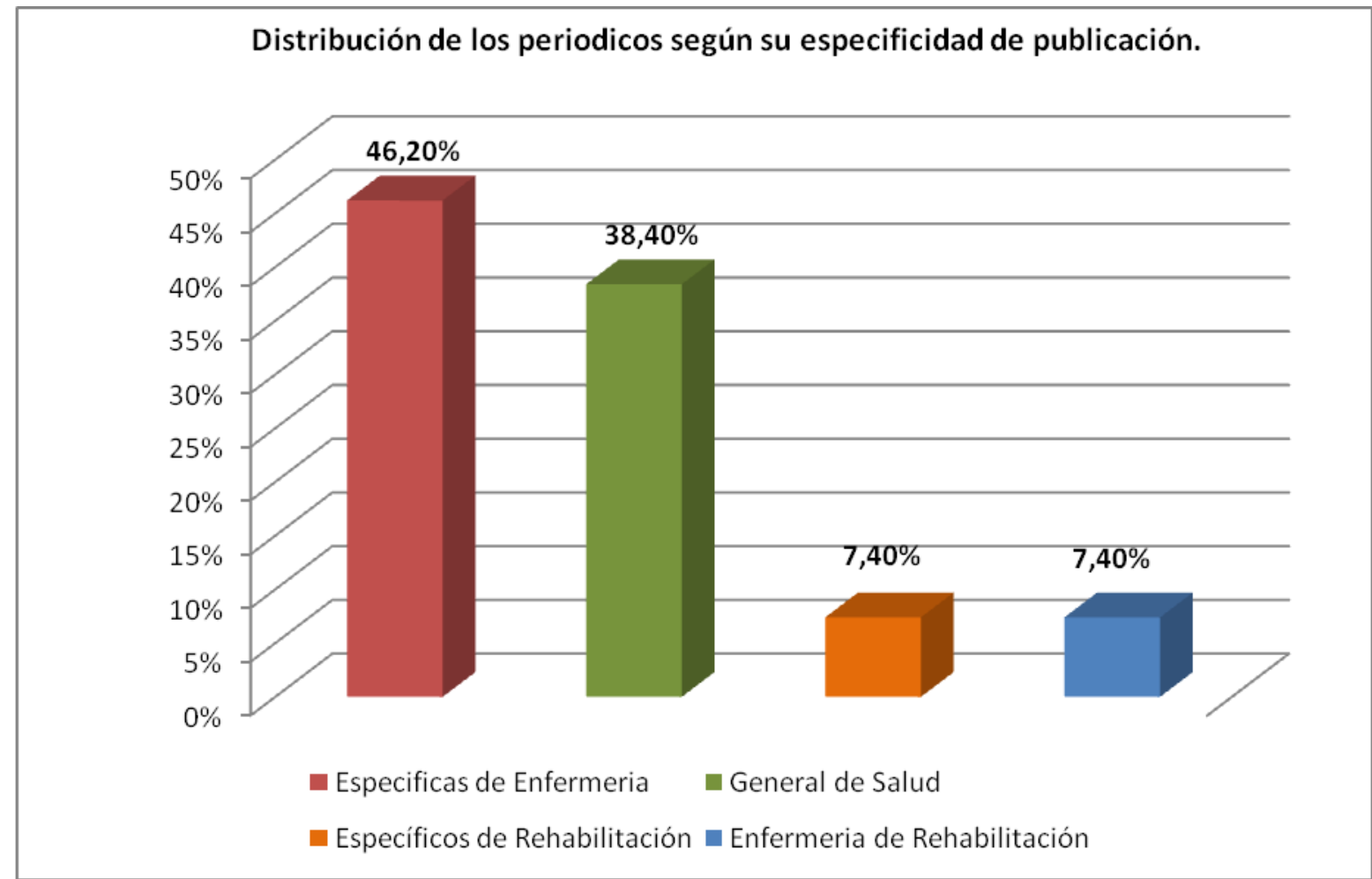

El Gráfico 3 muestra la relación de las categorías de profesionales que publicaron los artículos seleccionados para el estudio, en un total de 41 autores, resultando que en uno de los periódicos, cinco autores no informaron su formación académica y dos autores aparecen en más de una publicación.

[Gráfico 3. Distribución del porcentaje de autoría de los artículos según la categoría profesional.]

Distribución del porcentaje de outoría de los artículos según la categoría prefesional

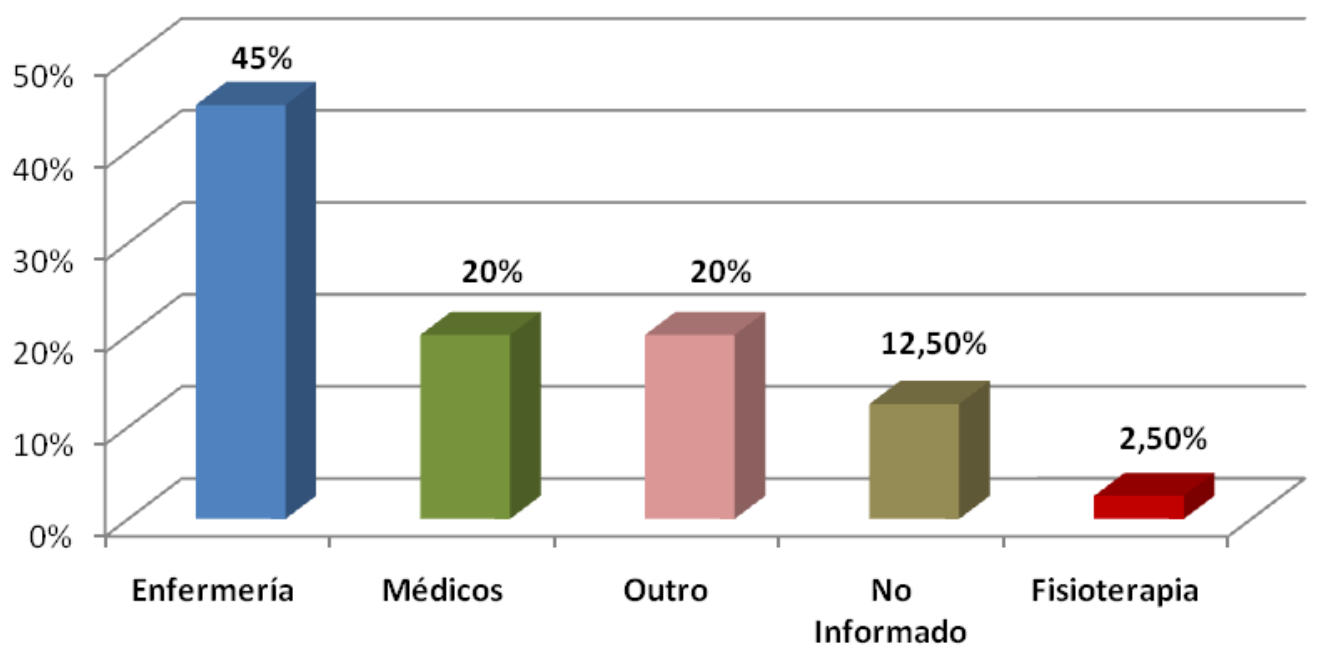

Con relación a la distribución por sexo de los autores de los artículos, se percibe que 22,5\% son de sexo masculino y $77,5 \%$ son del sexo femenino. 


\section{ASPECTOS HISTÓRICOS DE LA REHABILITACIÓN EN EL MUNDO}

A lo largo de la historia de la humanidad, se puede percibir que las personas portadoras de deficiencia sean motoras, sensoriales, mentales o de naturaleza congénita recibieron tratamiento diferenciado, de acuerdo con la cultura en que vivían. ${ }^{(5,6,7)}$.

Los hombres primitivos abandonaban esas personas, pues tenían la cultura de que solamente las "personas perfectas" deberían sobrevivir ${ }^{(6,8,9,10,11)}$.

En la antigüedad, periodo comprendido aproximadamente entre 4000 a.C a 395 d.C., ya se registraba preocupación con las personas que presentaban "diferencias incómodas". Había una preocupación por eliminar esas diferencias, por medio de recursos, técnicas, instrumentos $y / 0$ procedimientos ${ }^{(5,6,7,12,)}$.

En esta época no había estudios o aplicaciones en los cuales el interés estuviese volcado para evitar la ocurrencia de morbilidad ${ }^{(6)}$.

En 460 a.C., Hipócrates padre de la medicina, describió muchas deformidades y escribió en uno de sus libros "se debe tener en mente que el ejercicio fortalece y la inactividad enflaquece". Pudiéndose considerar así la base de la medicina física y de Rehabilitación. En esta misma época ya existían registros de utilización de miembros artificiales ${ }^{(13,14,15)}$.

Galeno, un médico romano que vivió aproximadamente entre 130 a 200 d.C., describió por primera vez en la Historia, la relación entre los huesos y los músculos del cuerpo, esa contribución está directamente relacionada con la rehabilitación física ${ }^{(6,13,15)}$.

Durante la Edad Media, periodo comprendido entre los siglos IV y XV, predominó la concepción, en todos los sectores de la sociedad de que la orden era establecida por el poder divino. En esta época la sociedad se organizaba jerárquicamente en clero, nobleza y camadas populares ${ }^{(6,14)}$.

La fé era una obligación de todos, se creía que todos los eventos naturales eran obra divina o del demonio. De esta forma ocurre una interrupción de los estudios de la medicina y sobre todo de la rehabilitación. Eso aconteció por dos motivos, el primero se dio por el hecho de que el cuerpo humano era considerado algo "inferior", con una valorización del culto al alma y al espíritu, y el segundo está relacionado con el clero y nobleza los mismos que estaban interesados en la actividad física, de modo que apenas hay aumento de la potencia física, ya que el ejercício no era más aplicado con miras a la cura. ${ }^{(6)}$.

En esta época los niños "defectuosos" dejan de ser abandonados, pues se creía que los factores religiosos interferían en las actitudes de las personas. Sin embargo, muchas personas fueron juzgadas y condenados por la inquisición, por ser "diferentes", eran tenidos como hechiceros y brujos ${ }^{(6,8,9)}$.

El periodo comprendido entre los siglos $\mathrm{XV}$ y XVI, llamado Renacimiento, hace que los rígidos patrones establecidos en la Edad Media entren en decadencia. Surgen las universidades y con eso la población busca la comprensión del Mundo y del conocimiento $(6,11,12,14,15)$

En esta época el Humanismo y las artes se desarrollan permitiendo el retorno de los estudios relativos a los cuidados con el cuerpo ${ }^{(6)}$. 
Mercurialis presenta los principios definidos como gimnástica médica que creía que los ejercicios eran importantes para conservar el estado de salud; es importante la regularidad en los ejercícios; los ejercicios pueden agravar, favorecer, ayudar y mejorar la calidad de vida de las personas sedentarias ${ }^{(6,7)}$.

La preocupación con la actividad física en este periodo no estaba solo ligada a los cuidados con el cuerpo, sino también con la conservación de las condiciones normales de los organismos considerados sanos ${ }^{(6,7)}$.

Don Francisco Y Ondeano Amorós, en 1849, divide la gimnástica en cuatro puntos, donde el tercero era La Cinesioterapia, que tenía como principio la conservación de la salud fuerte, tratamiento de las enfermedades, reeducación de los convalecientes y corrección de las deformidades ${ }^{(6)}$.

Estos nuevos conceptos permiten abarcar lo que hoy se denomina "promoción de la salud" además de la práctica curativa ${ }^{(6,7)}$.

Ya con la revolución industrial, periodo históricamente comprendido entre los siglos XVIII y XIX se inicia en Inglaterra un cambio en la sociedad, determinado por la producción en gran escala, mediante la utilización de máquinas, intensificando así el trabajo operario. De esta forma, surge el problema generado por la creciente utilización de maquinarias en las industrias, donde el número de accidentados de trabajo crece proporcionalmente ${ }^{(6,11,15)}$.

Las grandes y explotadoras jornadas de trabajo, condiciones sanitarias inapropiadas y condiciones de alimentación insatisfactoria, provocan la aparición y la proliferación de nuevas enfermedades. Eso hace que la medicina desarrolle estudios relacionados con este tipo de enfermedades $\left({ }^{6,7)}\right.$.

La medicina sufre una evolución dirigida a la atención del individuo enfermo, "medicina curativa y recuperativa", con la idea de la atención hospitalaria. De esta forma, la medicina de la era del surgimiento y desarrollo de la industia, pautada para el tratamiento de las enfermedades, deja de lado el camino que el Renacimiento traía, como la conservación de las condiciones satisfactorias de salud y prevención de enfermedades ${ }^{(6,7)}$.

Por ello, algunos estudiosos continuaron desarrollando el conocimiento en el área de la salud, como es el caso del médico británico, John Hunter, que en el siglo XVIII describió lo que se convirtió en la base de la reeducación de la musculatura, la relación entre la fuerza del paciente y amplitud del movimiento ${ }^{(6)}$.

En Alemania, en 1832, surge la primera escuela destinada a los deficientes físicos ${ }^{(6,16)}$.

En los siglos XIX y XX, comienzan a surgir las especializaciones, de forma organizada y más definida, en el sentido de compartir un área de estudio y campos de actuación profesional.

No interesaba la percepción global de los problemas incluyendo su red de determinantes, y sí las técnicas directas de "concierto" o de "rehabilitación" para que el individuo vuelva a una sociedad "integrada y productiva" (6).

En esta época los hospitales eran orientados también en el sentido de la especialización y concepto restringido de la cura, que atiende a la lesión o el problema que llevó al paciente a buscar al médico. Aun adecuadamente equipado y con especialidades habilitadas, un órgano continuaba deficiente en el sentido de restituir el hombre a la salud, en su concepto 
más amplio. El hospital no restituía al paciente de modo a restablecer el bienestar de su familia y de su grupo social ${ }^{(1)}$.

A pesar de todo, la lógica de la especialización profesional también produjo avances tecnológicos en muchos campos, aumentando la posibilidad de conocimiento y comprensión de la naturaleza de los fenómenos de las especializaciones estudiadas ${ }^{(6)}$.

Fue la necesidad de trabajo integral del paciente, lo que procuró la creación de la cuarta función de la medicina, después de la promoción, prevención y del tratamiento médico propiamente dicho, la rehabilitación ${ }^{(1)}$.

Se sabe que la Historia de la Rehabilitación está íntimamente ligada al crecimiento de una conciencia y responsabilidad social y que el interés mundial por esa especialidad ocurrió principalmente por cuatro acontecimientos históricos: las dos grandes Guerras Mundiales, el proceso acelerado de urbanización e industrialización, el favorecimiento de la propagación de epidemias y el aumento de accidentes de trabajo ${ }^{(2,13,17,18)}$.

En 1938, en un deseo por establecer normas y requisitos para la práctica de medicina de rehabilitación, se creó la Academia Americana de Medicina Física y Rehabilitación. Esa misma academia, en 1948, crea la Cámara Americana de Medicina Física y Rehabilitación (3).

Las dos grandes Guerras Mundiales hacen que el Mundo se depare por primera vez con el problema de un gran número de accidentados e incapacitados físicos. Personas que la mayoría de las veces eran jóvenes y con un futuro por vivir. De esta forma, se resalta la necesidad de restituir a esas personas en relación a las capacidades individuales y sociales $(8,9,18,19)$

Durante La II Guerra Mundial, la medicina comienza entonces a pensar en la necesidad de establecer un concepto para restaurar al enfermo en su potencial máximo, para así regresar a la sociedad, mejorando con eso sus condiciones en todas las esferas bio, psico, socio y espiritual $(1,2,8,9,18)$.

Frankyn Delano Roosevelt, parapléjico por poliomielitis, fue elegido presidente de los EUA, mostrando al Mundo la importancia de la rehabilitación para personas con deficiencia ${ }^{(11)}$.

En Holanda mucho antes de los registros de guerras, ya existía la Asociación de Fisioterapia y Sociedad de Fisioterapeutas Médicos. Pero el primer Centro de Rehabilitación, fue el Centro de Rehabilitación Militar, creado en la última fase de la Segunda Guerra Mundial, en 1944, con la intención de atender a los heridos de la Guerra. Pocos años más tarde, fue inagurado el primer Centro Civil de Rehabilitación también en este país, de acuerdo con los modelos americanos de la época. La medicina de rehabilitación en este pais está registrada como especialidad medica desde $1955^{(20)}$.

En Gran Bretaña, en la década de los 50, surge un servicio pionero, organizado por el gobierno, que se destina a la recuperación de deficientes, trabajadores de industrias, compuesto por 15 centros de Rehabilitación Industrial situados de Norte a Sur del país. En en un año cerca de 10 mil personas pasaron por esos centros, pudiendo la mayor parte de ellos retornar al mercado de trabajo ${ }^{(11,21)}$.

La década de 1970 a 1980 fue considerada la década de la rehabilitación, según decreto de la Asamblea de las Naciones Unidas. Fue también en la década de 80 cuando se percibió 
que la tecnología podría servir al hombre no solamente en la fase curativa y preventiva, sino también en la tercera fase de la medicina de rehabilitación ${ }^{(6)}$.

En las décadas de los 80 y 90 se utilizó en muchos países la Clasificación Internacional de las Desventajas (CIDID), desarrollada en 1976, después dio origen a la CIF - Clasificación Internacional de Deficiencias, traducida al portugués en 2003, se trata de un lenguaje universal, que es la funcionalidad ${ }^{(22,23,24)}$.

\section{ASPECTOS HISTÓRICOS DE LA REHABILITACIÓN EN EL BRASIL}

La rehabilitación en Brasil estuvo precedida de varios factores importantes. Está registrado que al final de siglo XIX, el emperador don Pedro II, preocupado con el problema de los ciegos, envió a Francia un médico, para que estudiara y trajese a nuestro país el conocimiento acerca de esta deficiencia. Como consecuencia surge el Instituo Benjamin Constant en Rio de Janeiro, para que proporcione atención a esos pacientes, el mismo que desarrolla sus actividades hasta el día de hoy ${ }^{(3)}$.

Durante el siglo XIX, con los avances de la medicina europea, manifestados también por la electroterapia e hidroterapia, despierta en el Brasil una tentativa de acompañar esas innovaciones. Así, en un periodo no muy preciso, entre 1879 y 1883, surge en Rio de Janeiro el primer gabinete de Electroterapia de América del Sur. Registros históricos muestran también, que en 1971 fue creada la Casa de las Duchas, donde se utilizaba agua dulce o del mar para tratamiento de las enfermedades ${ }^{(3)}$.

En1932, en Rio de Janeiro, fue creada la cátedra de Fisioterapia Médica, en la Facultad de Ciencias Médicas, la primera escuela de medicina en Brasil para enseñar Fisioterapia a sus estudiantes ${ }^{(3)}$.

Al terminar la Segunda Guerra Mundial, surge en los Estados Unidos y en Europa un movimiento para la organización de centros de rehabilitación, con la intención de rehabilitar soldados que sufrieron algún tipo de lesión física durante las batallas. A partir de ahí muchos médicos fueron a Estados Unidos y Europa, para adquirir conocimiento en esa nueva área, aún poco difundida en nuestro país. Es en este periodo que el desarrollo de los centros de rehabilitación se acentúa en Brasil y en el Mundo ${ }^{(19,25,26)}$.

No obstante, el surgimiento de instituciones de atención a las personas con deficiencia en Brasil, data de periodos históricos diferentes y adoptan modelos también diferentes de acuerdo con las circunstancias y agentes de su constitución, así como con el tipo de deficiencia que se destina para rehabilitar ${ }^{(25,27)}$.

Durante la gran epidemia de poliomielitis, se formaron los primeros centros de rehabilitación médica, para el tratamiento de secuelas de esta molestia ${ }^{(1,28)}$.

Antes de eso existían algunas instituciones, que trataban pacientes con alguna molestia crónica, con el objetivo de desarrollar la rehabilitación, o una de sus fases; es el caso del Hospital San Luis Gonzaga, que inició sus actividades en 1945, con un servicio de laborterapia, destinado a dar algunas ocupaciones a los pacientes tuberculosos ${ }^{(1)}$.

En1943, el Gobierno Nacional se manifiesta por primera vez sobre la causa de personas portadoras de enfermedades del aparato locomotor y en junio de este mismo año fue creado y aprobado la portaria 359 por el Ministerio de Educación y Salud de la época, denominándose Comisión de Atención a los Mutilados. En Mayo de 1944 la Portaria 260 del 
mismo Ministerio incorpora esa comisión a la División de Organización Hospitalaria, con el objetivo de poner en práctica un plan definitivo del servicio de atención a los mutilados ${ }^{(1)}$.

Por el decreto ley 5895 de 1943 quedó el Departamento Administrativo del Servicio Público (DASP) autorizado a aprovechar individuos con sus capacidades limitadas ${ }^{(1)}$.

Fue en 1947 cuando el primer Servicio de Medicina Física y Rehabilitación, en Rio de Janeiro, inicia sus actividades para ejecución de programas de rehabilitación médica ${ }^{(3)}$.

En 1946, Maria Hecilda Campos Salgados, después de cuidar a tres niños portadores de deficiencia en un refugio, funda la Casa Escuela San Francisco, con el objetivo de atender a niños portadores de deficiencias. En 1991 para optimizar el área disponible, fue firmado un convenio con la Escuela Paulista de Medicina - Universidad Federal de São Paulo ${ }^{(1,4)}$.

La literatura muestra que la primera clínica en recibir el nombre de Centro de Rehabilitación en Brasil, fue el Centro Piloto de Rehabilitación del SESI - Servicio Social de Industria, creado en agosto de 1950, en la ciudad de São Paulo, pero en esa ocasión existían registros de la Asociación de Atención a los Niños Deficientes - AACD, de carácter filantrópico, destinado a tratar niños portadores de secuelas de poliomielitis, parálisis cerebral o defectos congénitos ${ }^{(1,3,19)}$.

También en 1950 la Organización de las Naciones Unidas (ONU) dio inicio a la realización de una de sus metas más importantes, la implantación de un Instituto de Rehabilitación en un país de América Latina ${ }^{(1,19)}$.

Se optó por la ciudad de São Paulo para la instalación de este Instituto, porque en la época era una ciudad con gran demanda de personas que necesitaban de rehabilitación, pues poseía 29.000 fábricas y 840.000 operarios, además de contar con el Hospital de las Clínicas, un hospital escuela, con foco en la investigación, enseñanza y capacitación de sus profesionales $^{(1,19)}$.

En el año 1953, en la ciudad de São Paulo, se llevó a cabo el 1ํ Congreso Brasileño de Medicina Legal y Criminología, donde fue presentado un trabajo sobre la importacia económica y social de la rehabilitación en nuestro país y se debatió la posición de esta nueva especialidad en la instituciones de São Paulo ${ }^{(1,19)}$.

La mayor epidemia de poliomielitis de Rio de Janeiro, registrada en el año 1953, hizo que en 1954, un grupo de la sociedad carioca se empeñara en crear el primer centro de rahabilitación destinado a la atención de menores y adultos portadores de deficiencia física, con el objetivo de implantar y desarrollar la rehabilitación en su práctica, de una forma integrada, dentro de un moderno concepto definido por la Organización Mundial de la Salud como: aplicación de medidas médicas, sociales, educativas y profesionales; iniciando así sus actividades la Associação Beneficente de Reabilitação ${ }^{(2,19)}$.

Dos años después esa misma institución crea la Escuela de Rehabilitación de Rio de Janeiro, con el propósito de formar profesionales de nivel superior capacitados para atender esa demanda de pacientes ${ }^{(2,29)}$.

Fue también en 1954 cuando se fundó la Sociedad Brasileña de Fisioterapia que en 1959 pasa a ser designada Sociedad Brasileña de Medicina Física y Rehabilitación (2). 
En Bahia, Salvador, en 1958, fue creado un Centro de Rehabilitación, de origen particular. Simultáneamente surgieron en Minas Gerais y Pernambuco otros núcleos de rehabilitación $(1,3)$.

En 1956 se creó el Instituto Nacional de Rehabilitación - INAR, ya pensado desde 1950, recibiendo colaboración de la ONU y de la Organización Mundial de La Salud - OMS. Se destinaba esencialmente a ser un centro piloto de capacitación, formación de especialistas y también con finalidad de atención. En 1958 el INAR cambió su denominación por Instituto de Rehabilitación (IR) ${ }^{(1,19)}$.

En la década de los 60, de todas las Naciones de América del Sur, solo Brasil poseía un Centro de Rehabilitación integral, poseyendo los elementos humanos principales que constituye un equipo de rehabilitación integral y con posibilidad de atender todos los tipos de incapacitados físicos, independiente del sexo y edad ${ }^{(1)}$.

En noviembre de 1959, el Instituto de Rehabilitación organizó el primer Seminario sobre rehabilitación en Brasil. También en 1959, en Rio de Janeiro, surge el Instituto de Reeducación Motora, primer centro dedicado a la prevención de la paralisis cerebral y al tratamiento de niños de alto riesgo ${ }^{(1,19,26)}$.

En mayo de 1960, fue creado el Servicio de Rehabilitación del Departamento de profilaxia de la Lepra de la Secretaría de Salud del Estado de São Paulo ${ }^{(1,19,26)}$.

El servicio público de rehabilitación, llamado Sarah Kubistscheck, perteneciente a la Fundación de las Pioneras Sociales, fue creado en 1961, en Brasilia, DF. Hoy este centro Nacional tiene servicios instalados en diversos estados del Brasil ${ }^{(3)}$.

En el año 1970 es inagurada la División de Rehabilitación Profesional de Vergueiro, DRPV, perteneciente al Hospital de las Clínicas de la Facultad de Medicina de la Universidad de São Paulo, que en 1993 pasa a denominarse División de Medicina de Rehabilitación y, más recientemente, en enero de 2009, Instituto de Medicina Física y Rehabilitación - IMREA, incluyendo la Red Lucy Montouro ${ }^{(22)}$.

Entretanto la mayor parte de las Instituciones destinadas a rehabilitar, son creados por iniciativas particulares ${ }^{(1)}$.

La Fisiatria como especialidad médica, solo fue reconocida por la Asociación Médica Brasileña en 1972, donde el título de especialista pasó a ser fornecido por la Sociedad Brasileña de Medicina Física y Rehabilitación ${ }^{(3)}$.

Todos estos hechos históricos determinan el esenario actual de la rehabilitación en el Brasil, donde figuran diferentes instituciones con diferentes modelos de atención.

\section{LA ENFERMERÍA EN EL ESCENARIO DE LA REHABILITACIÓN EN BRASIL Y EN EL MUNDO}

En lo que se refiere a Enfermería, el papel educativo y rehabilitador del enfermero, son considerados desde el inicio de la Enfermería Moderna, cuando Florence Nightingale, en la Guerra de Crimea en 1859, prueba la eficiencia de las enfermeras capacitadas para la recuperación de los soldados heridos en la guerra ${ }^{(4,30)}$. 
Los registros de la literatura muestran que las primeras actuaciones de la enfermería, propiamente en rehabilitación, son de 1944, cuando el Sr. Ludwig Guttmann crea en Gran Bretaña el Centro de Traumatizados Medulares con el objetivo de normatizar el tratamiento de las lesiones medulares, así como la rehabilitación de esas personas en todo su contexto clínico y social ${ }^{(6)}$.

En 1920 con la Reforma Sanitaria fue creado el primer modelo de enfermería en Salud Publica, com el objetivo de que las enfermeras realicen visitas domiciliarias y educativas ${ }^{(31)}$.

Durante la II Guerra Mundial surge en Brasil la Fuerza Expedicionaria Brasileña (FEB), esta fuerza contaba en su formación con el Batallón de Salud, compuesto por 186 profesionales, entre ellos 67 enfermeras pioneras del ejército, con el objetivo de servir a esta institución mediante el cuidado de los brasileños heridos en la guerra ${ }^{(29)}$.

En 1980 surge la preocupación de delimitar el servicio desarrollado por el enfermero dentro de un Centro de Rehabilitación. Fue enviada una propuesta al Ministerio de Trabajo, para divulgar las actividades desarrolladas por este profesional, definida como Clasificación Brasileña de Ocupación de las actividades del(a) Enfermero(a) de rehabilitación, pero en esta década pocos eran los servicios existentes en Brasil para la rehabilitación de los deficientes físicos y raros eran aquellos que empleaban al profesional enfermero en su equipo de salud ${ }^{(32)}$.

Organizaciones internacionales desde la decada de los 80 estimulaban la formación y desarrollo de profesionales capacitados para la atención de personas portadoras de deficiencia, para que esas personas puedan integrarse en la comunidad de forma productiva, marcando con ello el despunte de la rehabilitación en Brasil con enfoque en el ser humano como un todo. Para conseguir ese objetivo, se hace necesario el profesional de enfermería dentro del equipo de rehabilitación, con su actuación en el área del autocuidado y educación para la salud ${ }^{(32,33,34,35)}$.

El proceso de atención al paciente en rehabilitación en la toda la Historia, desde su fase aguda, muestra la necesidad de trabajo en equipo interdisciplinar y transdisciplinar y la importancia del profesional de enfermería como parte integrante de ella ${ }^{(22,26,30,36)}$.

Es notable, a lo largo de los últimos 30 años, la expresión del enfermero en la rehabiliación de niños, adultos y ancianos con incapacidades y deficiencias, además de enfermedades crónicas. La investigación en el escenario nacional, sobre todo en la región sureste del Brasil, impulsó la formación de conocimientos propios para el enfermero rehabilitador, ya desarrollada en la práctica clinica de este especialista ${ }^{(22,26,36,37)}$.

Vale registrar que en 2002, en la Escuela de Enfermería de la Universidad de São Paulo, fue ofrecido el I Curso de Especialización de Enfermería en Rehabilitación, con la formación de 15 enfermeiro especialistas en la ciudad de São Paulo, configurándose como el primer curso de especialización de una universidad pública en Brasil. Desde 1994, la ex división de Medicina en Rehabilitación del Hospital de las Clínicas de la Facultad de Medicina de la Universidad de São Paulo, en la división de Enfermería ofrece el Curso de Perfeccionamiento de Enfermería en Rehabilitación ${ }^{(22,37)}$.

Barrel muestra la necesidad del profesional enfermero en la rehabilitación de pacientes con imcapacidades neurológicas y neuroquirúrgicas ${ }^{(38)}$. 
Es de esta forma que enfermería comienza a definir mejor su papel en este contexto, mostrando su importancia junto a esos servicios y procesos de rehabilitación.

\section{CONSIDERACIONES FINALES}

Conocer la Historia nos lleva a reflexionar y cuestionar sobre el contexto actual de la Rehabilitación, dirigida a la atención multidiciplinar y esencialmente holística del individuo y familia, como una ciencia de la inclusión social que lucha por medio de tratamientos especializados y del establecimiento de políticas sociales y de salud incluir el hecho y derecho de todas las personas que presentan deficiencias e incapacidades

Experiencias que vienen de las guerras y de las grandes epidemias muestran que la sociedad debe respetar las diferencias y trabajar de forma a conformar un mundo cada vez más inclusivo.

Fue a través de los cambios sociales, avances tecnológicos específicos y cambios políticos, educacionales, lo que permitió la organización de centros de rehabilitación y una conciencia social más sensible a la causa de las personas portadoras de deficiencias.

Es en este contexto histórico que nace la Medicina de Rehabilitación en el mundo y en Brasil, y la determinación del papel de la enfermera como fundamental en esta especialidad que tiene como principio el valor global del hombre.

Es necesario desarrollar ese raciocinio historico, para saber de donde venimos y cuál es el real objetivo que deseamos alcanzar con la evolución de la Rehabilitación

\section{REFERENCIAS BIBLIOGRÁFICAS}

1. Carvalho LF. Centro de reabilitação: casa do paraplégico. São Paulo: Reitoria da Universidade de São Paulo; 1963.

2.Barros FBM. Poliomielite, filantropia e fisioterapia: o nascimento da profissão de fisioterapeuta no Rio de Janeiro. Rev C S Col 2008; 13(3): 941-54.

03. Leitão REA. Medicina Física e Reabilitação. In: Leitão A, Leitão VA. Clínica de Reabilitação. São Paulo: Atheneu; 1995. p.1-12.

04. Nightingale F. Notas sobre Enfermagem. O que é e o que não é. São Paulo: Cortez; 1989.

05. Franco JR, Dias TRS. A pessoa cega no processo histórico: um breve percurso. [periódico na Internet]. São Paulo: Instituo Benjamin Constant. [acesso em 2009 Fev 10]. Disponível

em:

http://200.156.28.7/Nucleus/media/common/Nossos_Meios_RBC_RevAbr2005_Artigo\%201. doc

6. Rebelatto JR, Botomé SP. As alterações na concepção do objeto de trabalho em Fisioterapia em diferentes momentos de sua constituição. In: Rebelatto JR, Botomé SP. Fisioterapia no Brasil. $2^{a}$ ed. São Paulo: Manole; 1999. p.29-48.

07. Schewinsky SR. A barbárie do preconceito contra o deficiente - todos somos vitimas. Acta Fisiátrica. 2004; 11(1): 7-11.

08. Pessini L, Ferrari MAC, Gonçalves MJ. Reabilitação: de um olhar histórico aos desafios contemporâneos. O Mundo da Saúde. 2008; 30(21): 5-9.

09. Pessini L, Ferrari MAC, Gonçalves MJ, Lucareli PRG. Reabilitação Múltiplo enfoques. O Mundo da Saúde. 2008; 32(2).

10. Silva OM. A epopéia ignorada: a pessoa deficiente na história do mundo de ontem e de hoje. São Paulo: CEDAS; 1986. 
11. Pacheco KMB, Alves VLR. A História de deficiência, da marginalização à inclusão social: uma mudança de paradigma. Acta Fisiátrica. 2007; 14(4): 242-8.

12. Rodrigues G. Um olhar para além da deficiência [periódico na Internet]. São Paulo:

Pedago Brasil. [cesso em 2009 Fev 10]. Disponível em: http://www.pedagobrasil.com.br/educacaoespecial/umolharparaalemdasdeficiencias.htm

13. Boroch RM. Elements of rehabilitation in nursing: an introduction. Saint Louis: Mosby;1976.

14. Grieco FS, Pinto AS, Garcia GR, Paiva RP. Evoucion histórica de La traumatologia y ortopedia. Gac. Méd. Caracas. 1999; 107 (2): 280-7.

15. Cherem AJ. Medicina e arte: observações para um dialogo interdisciplinar. Acta Fisiátrica. 2005; 12(1): 26-32.

16. Camargo SM. Um breve panorama do que já foi feito pela educação no Brasil e no mundo. [periódico na Internet]. São Paulo: Pedago Brasil. [acesso em 2009 Fev 10]. Disponível em: http://www.pedagobrasil.com.br/educacaoespecial/umbrevepanorama.htm

17. Tittanegro CR. Aspectos filosóficos da reabilitação. O Mundo da Saúde. 2006; 30(1): 20 5.

18. Arruda L. Centro de Medicina de Reabilitação do Alcoitão: história do projeto de reabilitação; a arquitetura e a arte. Lisboa; 2006.

19. Carvalho LF. Reabilitação dos deficientes físicos no Brasil. Rev. Paulista de Hospitais. 1963; 7(5): 11-7.

20. Peek WJ. A história da medicina de reabilitação como especialidade médica na Holanda. Acta Fisiátrica. 1998; 5(3): 170-2.

21. A reabilitação nos centros industriais da Grã-Bretanha. Rev. Paulista de Hospitais. 1959; 7(3):36.

22. Faro ACM. Enfermagem em reabilitação: ampliando os horizontes, legitimando o saber.

Rev.Esc.enferm.USP. 2006; 40(1): 128-33.

23. Amiralian MLT, Pinto EB, Ghirardi MIG, Lichtig I, Masini EFS, Pasqualin L. Conceituando deficiência. Rev. Saúde Pública. 2000; 34(1): 97-103.

24. Buchalla CM. CIF: classificação internacional de funcionalidade, incapacidade e saúde. Centro Colaborador da Organização Mundial da Saúde para a família de Classificações Internacionais. São Paulo: Universidade de São Paulo; 2003.

25. Alves VLR. O significado do discurso de risco na área de reabilitação. Acta Fisiátrica. 2001; 8(2): 67-70.

26. Mancussi AC. Assistência ao binômio paciente/família na situação de lesão traumáticada medula espinhal. Rev. Latino-Am. Enfermagem. 1998; 6(4):67-73.

27. Santos VLCG, Miyadahira AMK, Kimura M. Assistência em reabilitação: um processo marcado por deficiências. Rev. Gaúcha de Enferm. 1990; 11(2): 5-11.

28. Battistella L. Interfases da Medicina de reabilitação com a medicina do trabalho e as ciências Forenses. Acta Fisiátrica. 2003; 10(2): 52-3.

29. Santos TCF, Lopes GT, Bernardes MMR. Bases de sustentação militar de Vargas durante a $2^{\circ}$ guerra e soberania bélica alemã: percepções de enfermeiras militares. Texto contexto - enferm. 2005; 14(4):344-50.

30. Potter PA, Perry AG. A Enfermagem Atual. In: Potter PA, Perry AG, organizadores. Fundamentos de Enfermagem. 6aㅡ. ed. Rio de Janeiro: Elsevier; 2006. p.3-7.

31. Barreira IA. A pratica da enfermagem no Brasil: a enfermeira de saúde publica dos anos 20. Texto contexto - enferm. 1998; 7(1): 42-57.

32. Comaru MN, Santos WLR, Neves TA, Donadel NA. Subsídios para determinação das atividades do(a) enfermeiro(a) de reabilitação. Rev. bras. enferm. 1980; 33:173-189.

33. Diogo MJD. O papel da enfermeira na reabilitação do idoso. Rev. Latino-Am. Enfermagem. 2000; 8(1): 75-81.

34. Comarú MN, Neves TA, Kerlesz PM. Participação da(o) enfermeira (o) num programa de reabilitação - relato de experiência. Rev. bras. enferm. 1978; 31: 237-43. 
35. Gomes BP. Contributos da formação para o desenvolvimento de competências na área da saúde de reabilitação. Texto contexto - enferm. 2006; 15(2): 193-204.

36. Delisa JA, Currie DM, Martin GM. Medicina de Reabilitação: passado, presente e futuro. In: Delisa JA, Gans BM, organizadores. Tratado de Medicina de Reabilitação. Princípios e Práticas. $3^{\mathrm{a}}$ ed. Barueri SP: Manole; 2002. p.3-33.

37. Tucker NJ. Geriatric rehabilitation: Nursing Challenge of the'90s. Rehabilitation Nursing. 1993; 18(2): 114-6.

38. Borrel MA, Ferrer RM, Sotomayor DO. Papel de la enfermera em la reabilitacion del paciente neurológico y neuroquirurgico. Rev Cubana Enfermer 1994; 10(2): 90-6. 
ANEXO 1. Catálogo de las Publicaciones.

\section{Sobre la Fuente:}

Data da publicación:

Documento:

Tema Abordado:

Fuente:

Pais de origem:

Periodicidad:

Categoría: General ( ) Específica ( ) Cuál :

$\mathrm{n}^{\circ}$ de artículos:

Observación:

\section{Sobre los autores:}

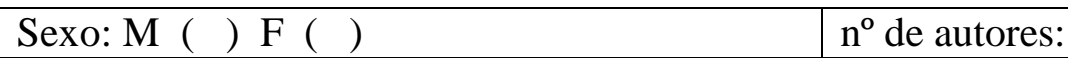

Formación académica: Enfermero ( ) Médico ( ) Fisioterapeuta ( ) Otro ( ) cuál:

Nacionalidad: Investigación financiada: $\mathrm{s}(\quad) \mathrm{N}(\quad) \sin$ información ( )

Instituición de Origem:

Instituición de trabajo: Hospital ( ) Cuál?:

Universidad ( ) Cuál?:

Institución ( ) Cuál?

Titulación: Magister ( ) Doctor ( ) Libre docente ( ) Especialista

Prof Titular ( ) Universidad:

Docente Adjunto:

\section{Sobre el texto:}

Objetivo: 1

2:

3:

Metodología:

Tipo de investigación:

Descriptores: 1:___ 2

3 .

4:

Instrumentos:

OBS: 
ISSN 1695-6141

(c) COPYRIGHT Servicio de Publicaciones - Universidad de Murcia 